\title{
PIK3R1 gene polymorphisms are associated with type 2 diabetes and related features in the Turkish population
}

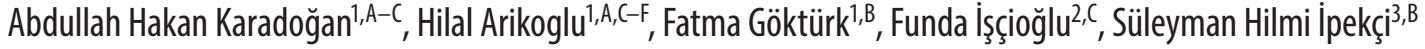 \\ 1 Department of Medical Biology, Faculty of Medicine, Selçuk University, Konya, Turkey \\ 2 Department of Statistics, Faculty of Science, Ege University, Izmir, Turkey \\ ${ }^{3}$ Department of Endocrinology, Faculty of Medicine, Selçuk University, Konya, Turkey \\ A - research concept and design; B - collection and/or assembly of data; $\mathrm{C}$ - data analysis and interpretation; \\ $\mathrm{D}$ - writing the article; $\mathrm{E}$ - critical revision of the article; $\mathrm{F}$ - final approval of the article
}

\section{Address for correspondence}

Hilal Arikoglu

E-mail: harikoglu@selcuk.edu.tr

Funding sources

This study was supported by the Selçuk University Research Foundation (13202033).

Conflict of interest

None declared

\section{Acknowledgements}

We would like to thank Dr. Sevim Karakas

for her assistance in this study.

Received on February 22, 2016

Reviewed on July 22, 2016

Accepted on February 14, 2017

\begin{abstract}
Background. The phosphatidylinositol 3-kinase p85 alpha regulatory subunit 1 gene (PIK3R1) encodes the PIK3R1 protein, which plays a direct role in insulin signaling. PIK3R1 (p85 regulatory subunit) connects firmly with the p110 catalytic subunit, and together these proteins form the phosphatidylinositol 3-kinase (PI3K) protein. PI3K is a key protein in the Akt signaling pathway, which regulates cell survival, growth, differentiation, glucose trafficking, and utilization. Defects in the insulin signaling cascade play an important role in the development of insulin resistance, which shares a common genetic basis for metabolic diseases such as type 2 diabetes (T2D), obesity and cardiovascular diseases.
\end{abstract}

Objectives. In our study, we investigated the effect of single nucleotide polymorphisms (SNPS) rs3756668 in $3^{\prime} U T R$ region, rs706713 and rs3730089 in exons 1 and 6 , respectively, rs7713645 and rs7709243 in intron 1 , and rs1550805 in intron 6 of PIK3R1 gene on T2D.

Material and methods. This study enrolled a total of 840 individuals, including 427 diabetic individuals (206 obese and 221 non-obese) and 413 nondiabetic individuals (138 obese and 275 non-obese). The target SNPs were analyzed using real-time polymerase chain reaction (RT-PCR). Statistical analysis was performed using SPSS18.0 (IBM Corp., Armonk, USA). The p-values $\leq 0.05$ were considered statistically significant.

Results. The SNPs rs706713 (Tyr73Tyr) and rs3730089 (Met3266le) located in exons, and rs7713645, rs7709243 and rs1550805 located in introns were determined to be significantly associated with T2D and phenotypic features such as obesity, insulin resistance and the lipid parameters. The association with SNP rs3756668, which is located in the 3'UTR, was not significant.

Conclusions. Our study supports the role of PIK3RT, an important candidate gene due to its critical role in insulin signal transduction, in T2D development.

Key words: type 2 diabetes, single nucleotide polymorphisms, PIK3RT gene

DOI

10.17219/acem/68985

Copyright

Copyright by Author(s)

This is an article distributed under the terms of the

Creative Commons Attribution Non-Commercial License

(http://creativecommons.org/licenses/by-nc-nd/4.0/) 


\section{Introduction}

Insulin is a major hormone that regulates processes such as cellular growth, differentiation and apoptosis, in addition to glucose metabolism. Insulin acts on target tissues, mainly liver, muscle and adipose tissues, by binding to its receptor on the cell surface, leading to the autoactivation of tyrosine residues. Once activated, receptor phosphorylates the downstream signaling proteins known as the insulin receptor substrate (IRS) family, which has Src Homology 2 (SH2) domain-containing proteins. Then, phosphorylated IRS proteins activate other SH2 domaincontaining proteins, including the regulatory subunits of phosphatidylinositol 3-kinase (PI3K). PI3K catalyzes the conversion of phosphatidylinositol phosphate (PIP) 2 to PIP3, which activate Akt signaling, which itself regulates cell survival, growth, differentiation, glucose transporter type 4 (GLUT-4) trafficking, and glucose utilization. ${ }^{1}$

Impaired activation of the insulin signaling pathway results in the decreased responsiveness of target tissues to normal circulating levels of insulin, a condition known as insulin resistance. Insulin resistance is a common pathogenesis in several metabolic diseases, such as type 2 diabetes (T2D), obesity and cardiovascular diseases.

Insulin resistance generally occurs as a result of postreceptor defects. ${ }^{2}$ Especially, it is suggested that reductions in both IRS-l phosphorylation and insulin-stimulated PI3K activation leads to impaired insulin signal transduction. Additionally, other signaling molecules in this transduction, insulin signaling inhibitors such as ENPP1, PTP1B, and INPPL1, lipid accumulation in target tissues, and abnormal lipid metabolism can also contribute to insulin resistance. $^{3,4}$

The PI3K enzyme is composed of 2 subunits, the p110 catalytic subunit and regulatory p $85 \alpha$ subunit. ${ }^{5}$ The $\mathrm{p} 85 \alpha$ subunit stabilizes the p110 catalytic subunit and determines the level of activity. ${ }^{5}$ Polymorphisms in the PIK3R 1 gene, which encodes the 855 regulatory subunit of the PI3K enzyme, are associated with the development of diseases such as insulin resistance, obesity, T2D, and cancer. ${ }^{6-8}$ In this study, we investigated the effects of single nucleotide polymorphisms (SNPs) rs3756668A $\rightarrow$ G (located in the 3'UTR region), $r 5706713 \mathrm{C} \rightarrow \mathrm{T}$ and $\mathrm{rs} 3730089 \mathrm{~A} \rightarrow \mathrm{G}$ (located in exons 1 and 6, respectively), rs7713645A $\rightarrow \mathrm{C}$ and rs7709243C $\rightarrow \mathrm{T}$ (both located in intron 1), and rs1550805C $\rightarrow \mathrm{T}$ (located in intron 6) of the PIK3R1 gene on T2D.

\section{Material and methods}

\section{Clinical samples}

This study enrolled a total of 840 individuals, including 427 diabetic individuals (206 obese and 221 non-obese) and 413 nondiabetic individuals ( 138 obese and 275 non-obese). These individuals were selected from patients admitted to the Selçuk University Hospital, Department of Endocrinology (Konya, Turkey). The individuals in the diabetic group, who were diagnosed according to the guidelines of the American Diabetes Association (ADA), were over 30 years old and did not use insulin. Other types of diabetes (type 1 diabetes, maturity onset diabetes of the young - MODY, latent autoimmune diabetes of adults - LADA, drug-related and other types) were excluded. Obesity criteria was considered as body mass index $(\mathrm{BMI}) \geq 30$. The control group in this study included age- and BMI-matched nondiabetic individuals with no family history of diabetes. Written informed consent was obtained from each individual before participation in the study. The study was approved by the Ethics Committee of the Selçuk University Medical Faculty (Konya, Turkey). An oral glucose tolerance test (OGTT) was performed on the individuals considered as pre-diabetic. Individuals with impaired glucose tolerance or insulin resistance were excluded from the study.

\section{Clinical analyses}

Fasting plasma glucose, fasting insulin, HbA1C, c-peptide, cholesterol, low density lipoprotein (LDL), high density lipoprotein (HDL), and triglyceride values were measured for both diabetic and control groups. Insulin resistance was determined using the homeostasis model assessment of insulin resistance (HOMA-IR) and was calculated as fasting plasma glucose $(\mathrm{mmol} / \mathrm{L})$ multiplied by fasting serum insulin (pmol/L) and divided by 22.5 . An individual with a HOMA-IR value higher than 2.5 was considered resistant to insulin.

\section{Genotyping}

Genomic DNA was isolated from peripheral blood leukocytes using a standard proteinase K and sodium dodecyl sulphate (SDS) procedure. Target SNPs were analyzed using the real-time polymerase chain reaction (RT-PCR) technique and a TIB MolBiol LightSNiP kit (Roche, Basel, Switzerland). Six SNPs in the PIK3R1 gene were previously reported to be associated with the disease in the literature. Information about these target SNPs is shown in Table 1.

\section{Statistical analysis}

Descriptive statistics were obtained for clinical and biochemical characteristics. Normally distributed parameters were compared using one-way analysis of variance (ANOVA), while parameters that did not show normal distributions were compared by Kruskal-Wallis test after transformation.

The differences between the patient and control groups in terms of genotype distribution or allele frequency were analyzed using Pearson's $\chi^{2}$ test. A $\chi^{2}$ goodness of fit test was used to evaluate Hardy-Weinberg equilibrium (HWE) 
Table 1. Clinical and biochemical characteristics of study subjects

\begin{tabular}{|c|c|c|c|c|c|c|c|c|}
\hline \multirow[b]{2}{*}{ Variable } & \multicolumn{2}{|c|}{ Obese } & \multicolumn{2}{|c|}{ Non-obese } & \multicolumn{4}{|c|}{$p$-value } \\
\hline & $\begin{array}{l}\text { DM } \\
(1)\end{array}$ & $\begin{array}{l}\text { control } \\
\text { (2) }\end{array}$ & $\begin{array}{l}\text { DM } \\
(3)\end{array}$ & $\begin{array}{l}\text { control } \\
(4)\end{array}$ & $p(1-2)$ & $p(1-3)$ & $p(2-4)$ & $p(3-4)$ \\
\hline Number (840) & 206 & 138 & 221 & 275 & NA & NA & NA & NA \\
\hline Age [years] & $55.6 \pm 8.8$ & $48.9 \pm 11.3$ & $56.5 \pm 9.3$ & $43.9 \pm 10.4$ & $<0.001^{*}$ & 0.792 & $<0.001^{*}$ & $<0.001^{*}$ \\
\hline Gender (M/F) & $154 / 52$ & $120 / 18$ & $86 / 135$ & 182/93 & NA & NA & NA & NA \\
\hline BMI $\left[\mathrm{kg} / \mathrm{m}^{2}\right]$ & $35.14 \pm 4.23$ & $34.57 \pm 3.92$ & $26.74 \pm 2.74$ & $26.08 \pm 3.01$ & 0.445 & NA & NA & 0.162 \\
\hline Insulin' $[\mu \mid \bigcup / m L]$ & $\begin{array}{c}11.89 \\
(2.04-40.79)\end{array}$ & $\begin{array}{c}10.85 \\
(4.31-39.98)\end{array}$ & $\begin{array}{c}7.90 \\
(1.00-37.30)\end{array}$ & $\begin{array}{c}7.33 \\
(2.00-39.91)\end{array}$ & 0.270 & $<0.001^{*}$ & $<0.001^{*}$ & 0.297 \\
\hline C-peptide $[\mathrm{ng} / \mathrm{mL}]$ & $\begin{array}{c}3.07 \\
(0.74-9.40)\end{array}$ & $\begin{array}{c}2.51 \\
(1.49-6.30)\end{array}$ & $\begin{array}{c}2.00 \\
(0.50-16.10)\end{array}$ & $\begin{array}{c}2.13 \\
(0.70-15.70)\end{array}$ & 0.292 & $<0.001^{*}$ & 0.013 & 0.067 \\
\hline $\mathrm{FG}^{1}[\mathrm{mg} / \mathrm{dL}]$ & $\begin{array}{c}140 \\
(64-377)\end{array}$ & $\begin{array}{c}94 \\
(76-113)\end{array}$ & $\begin{array}{c}134 \\
(59-363)\end{array}$ & $\begin{array}{c}92 \\
(58-114)\end{array}$ & $<0.001^{*}$ & 0.548 & $0.001^{*}$ & $<0.001^{*}$ \\
\hline $\operatorname{Alc}^{1}(\%)$ & $\begin{array}{c}7.1 \\
(5.2-12.5)\end{array}$ & $\begin{array}{c}5.6 \\
(5.3-6.4)\end{array}$ & $\begin{array}{c}7.1 \\
(5.1-19.5)\end{array}$ & $\begin{array}{c}5.5 \\
(5.0-6.4)\end{array}$ & $<0.001^{*}$ & 0.915 & 0.212 & $<0.001^{*}$ \\
\hline $\mathrm{TG}^{1}[\mathrm{mg} / \mathrm{dL}]$ & $\begin{array}{c}171 \\
(59-674)\end{array}$ & $\begin{array}{c}126 \\
(52-380)\end{array}$ & $\begin{array}{c}128 \\
(41-457)\end{array}$ & $\begin{array}{c}116 \\
(40-478)\end{array}$ & $<0.001^{*}$ & $0.002^{*}$ & 0.029 & 0.055 \\
\hline Chol [mg/dL] & $203 \pm 45$ & $211 \pm 39$ & $197 \pm 47$ & $202 \pm 42$ & 0.415 & 0.632 & 0.332 & 0.817 \\
\hline $\mathrm{LDL}^{1}[\mathrm{mg} / \mathrm{dL}]$ & $\begin{array}{c}122 \\
(52-276)\end{array}$ & $\begin{array}{c}130 \\
(78-239)\end{array}$ & $\begin{array}{c}124 \\
(35-271)\end{array}$ & $\begin{array}{c}125 \\
(39-294)\end{array}$ & $0.006^{*}$ & 0.929 & 0.169 & 0.257 \\
\hline $\mathrm{HDL}[\mathrm{mg} / \mathrm{dL}]$ & $43 \pm 10$ & $47 \pm 11$ & $44 \pm 13$ & $47 \pm 12$ & $0.003^{*}$ & 0.786 & 0.991 & 0.055 \\
\hline HOMA-IR 1 & $\begin{array}{c}4.42 \\
(0.79-18.00)\end{array}$ & $\begin{array}{c}2.61 \\
(0.83-9.90)\end{array}$ & $\begin{array}{c}2.80 \\
(0.42-17.00)\end{array}$ & $\begin{array}{c}1.67 \\
(0.40-12.00)\end{array}$ & $<0.001^{*}$ & $<0.001^{*}$ & $<0.001^{*}$ & $<0.001^{*}$ \\
\hline
\end{tabular}

${ }^{1}$ Non-normally distributed parameters are shown as median (min-max); ${ }^{*}$ statistically significant differences were found; NA - this data could not be evaluated for statistical significance because it was not found to be clinically significant. In post-hoc test, $\mathrm{p}$-value $=0.008$ was considered statistically significant for comparing 4 groups; DM - diabetes mellitus; FG - fasting glucose; TG - triglyceride; Chol - cholesterol; LDL - low-density lipoprotein; HDL - high-density lipoprotein; HOMA-IR - homeostasis model assessment of insulin resistance.

in the patient and control groups. Analyses were performed using dominant, additive and recessive models. Dominance was defined in terms of allele 2 effects. In dominant allele 2 models, homozygous individuals for allele 1 were compared with carriers of allele 2 . In recessive allele 2 models, homozygous individuals for allele 2 were compared with carriers of allele 1. Association between genotypes and T2D was tested by conducting a case-control study. Allele frequencies of SNPs in the patient and control groups were evaluated using odds ratio (OR). We performed logistic regression analysis by considering not only dominant, additive and recessive models of SNP genotypes, but also sex and age.

We also investigated if there was any association between genotypes and biochemical characteristics of T2D. Therefore, we performed tests to determine the normality of the biochemical characteristics. Fasting plasma insulin and triglyceride variables were normalized by log transformation, and cholesterol was normalized by square root transformation, before ANOVA analysis. For the other characteristics (BMI, fasting glucose, c-peptide, HbA1C, LDL, HDL, and HOMA-IR), the Kruskal-Wallis test was performed. The SNP genotypes were coded as 11, 12 and 22. Then, a single point (single SNP) regression analysis model was fitted. Linkage disequilibrium (LD) analysis was performed with MIDAS software. ${ }^{9}$
All analyses were carried out using SPSS 18.0 (IBM Corp., Armonk, USA). In all analyses, $\mathrm{p}$-value $\leq 0.05$ was considered statistically significant.

\section{Results}

\section{Characteristics of study subjects}

A total of 840 individuals, including 427 diabetic individuals (206 obese and 221 non-obese) and 413 nondiabetic individuals (138 obese and 275 non-obese), were enrolled in this study. The clinical and biochemical characteristics of the individuals studied are shown in Table 2.

\section{Association study}

The genotype distribution and association analyses of SNPs are summarized in Table 3. The silent SNP rs706713 in exon 1 of the PIK3R 1 gene showed a significant association with T2D (OR 1.7 (95\% confidence interval CI: 1.09-2.40), $\mathrm{p}=0.016$ and OR 1.5 (95\% CI: 1.054-2.233), $\mathrm{p}=0.025$, additive and dominant models, respectively) when individuals were considered as patient and control groups. When the obese and non-obese groups were evaluated separately, a strong relationship between SNP 
Table 2. Genotype distribution of studied SNPS in the PIK3R1 gene and their association analysis results in diabetics and controls

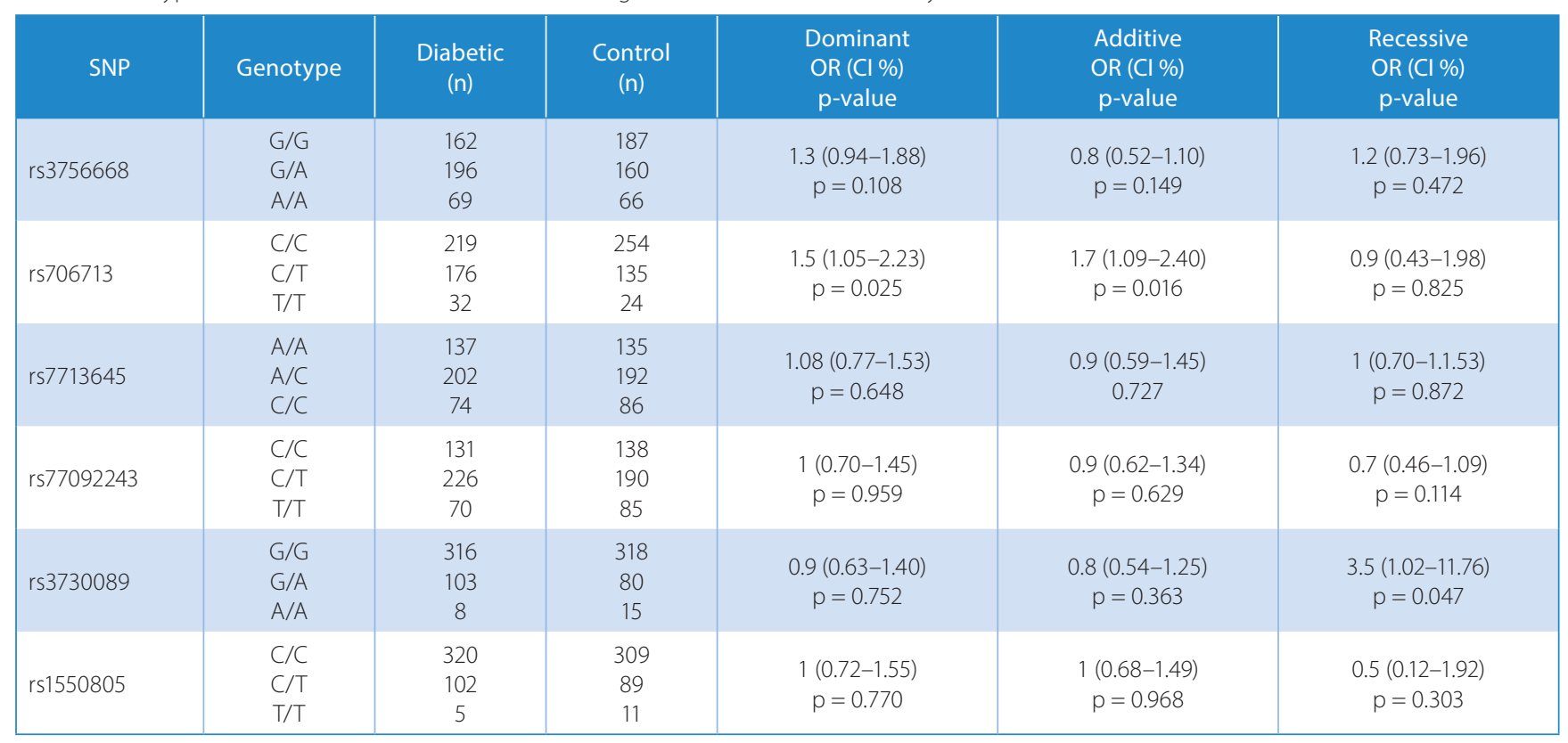

SNPs - single nucleotide polymorphisms; OR - odds ratio; Cl - confidence interval.

Table 3. Genotype distribution of studied SNPs in the PIK3R1 gene and their association analysis results in obese and non-obese diabetics and controls

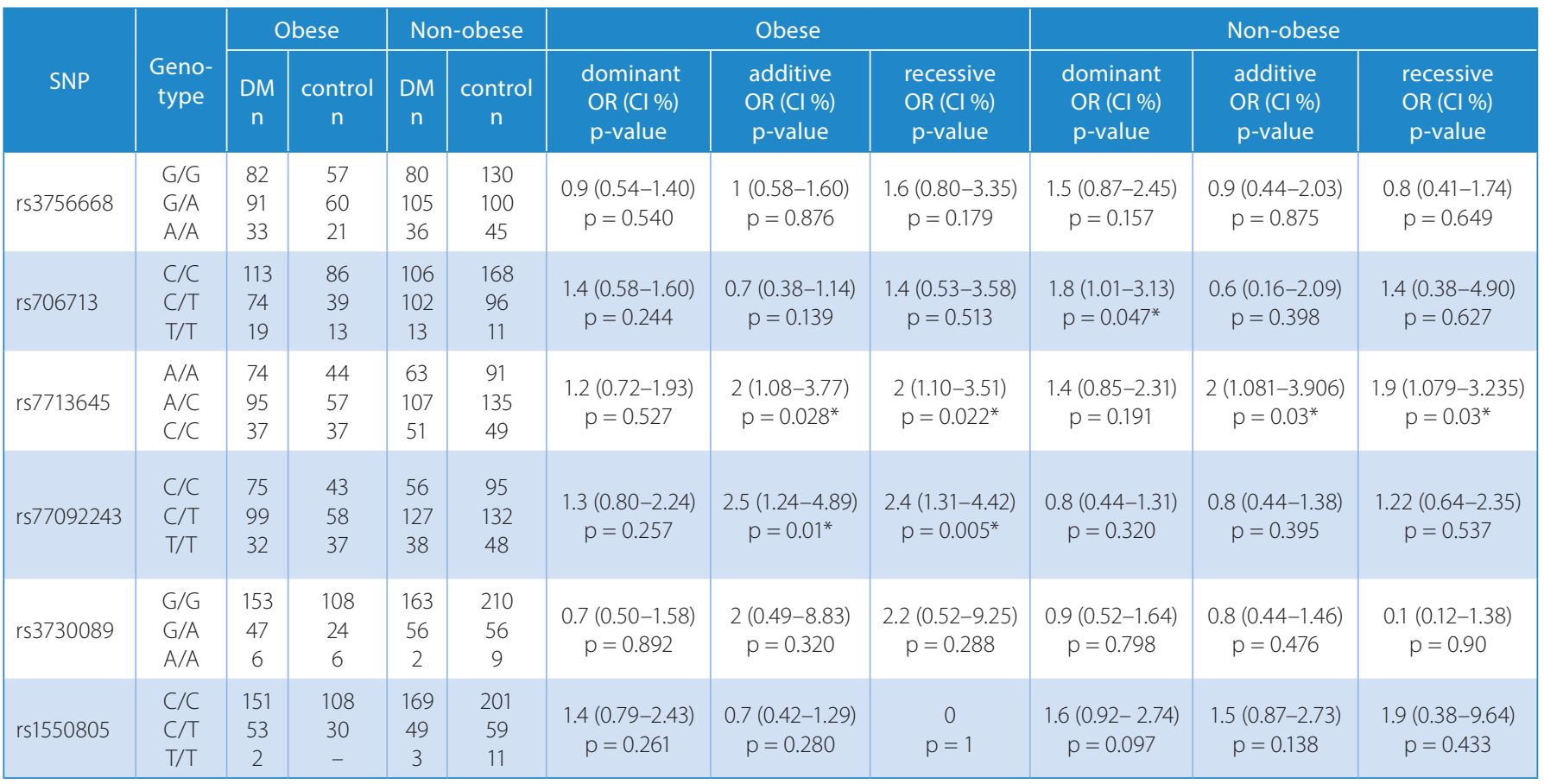

SNP - single nucleotide polymorphism; DM - diabetes mellitus; OR - odds ratio; $\mathrm{Cl}$ - confidence interval; ${ }^{*}$ statistically significant.

rs706713 and T2D was also identified in the non-obese group (OR 1.8 (95\% CI: 1.006-3.128), p = 0.05, dominant model). The groups were in HWE for this SNP when considered either together or separately $(\mathrm{p}>0.05)$.

The SNP rs3730089 in exon 6 causes an amino acid substitution in the protein, and was significantly associated with T2D (OR 3.5 (95\% CI: 1.015-11.762), p = 0.047, recessive model). The genotype distribution for this SNP was found to deviate from HWE in the control groups ( $\mathrm{p}<0.05)$.

While the intronic SNP rs7709243 was associated with T2D in the obese group (OR 2.5 (95\% CI: 1.239-4.891), $\mathrm{p}=0.01$ and OR 2.4 (95\% CI: 1.309-4.418), $\mathrm{p}=0.005$, additive and recessive models, respectively), the rs7713645 SNP was associated with the disease in both the obese group (OR 2 (95\% CI: 1.081-3.773), $\mathrm{p}=0.03$ and OR 2 (95\% CI: 1.101-3.514), $\mathrm{p}=0.022$, additive and recessive models, respectively) and the non-obese group (OR 2 (95\% CI: 1.081-3.906), $\mathrm{p}=0.03$ and OR 1.9 (95\% CI: 1.079-3.235), $\mathrm{p}=0.03$, additive and recessive models, respectively). With the exception of the genotype distribution of SNP rs7709243 in the non-obese diabetic group, both of the SNPs located in intron 1 were in HWE. 
Neither the SNP rs1550805 in intron 6 nor the SNP rs3756668 in the 3'UTR of the PIK3R1 gene were associated with $\mathrm{T} 2 \mathrm{D}(\mathrm{p}>0.05)$ when the groups were assessed together or separately. The genotype distributions of both SNPs showed deviation from HWE in the control groups.

Additionally, linkage disequilibrium was calculated for the 6 SNPs and $r^{2}$ values were used to provide evidence of pairwise LD between the SNPs. Only the rs7709243 and rs7713645 SNPs located in intron 1 were found to be in strong $\mathrm{LD}\left(\mathrm{D}^{\prime}=0.85 ; \mathrm{r}^{2}=0.7\right)$. The LD between the other variants was weak.

\section{Genotype-phenotype relationship}

The effects of the SNPs on phenotypic characteristics such as fasting glucose, insulin, c-peptide, hemoglobin A1C levels, triglycerides, cholesterol, LDL and HDL levels, BMI, and HOMA-IR value as an insulin resistance marker were analyzed in this study. The SNP rs706713 in exon 1 had a strong effect on HOMA-IR $(\mathrm{p}=0.018)$. The intronic SNP rs7709243 showed an effect on LDL level $(p=0.028)$ and cholesterol ( $p=0.008)$, while the other intronic SNP, rs1550805, had a strong effect on c-peptide level $(p=0.023)$ and BMI ( $=0.007)$.

\section{Discussion}

Insulin has both metabolic and growth-stimulating effects, and its metabolic effect occurs through the PI3K/Akt pathway. ${ }^{10}$ Although other growth factors also activate PI3K via distinct receptor tyrosine kinases, PI3K activation, which occurs only through insulin effect, has been found to regulate metabolic homeostasis. ${ }^{11}$ In adipocyte cell cultures, it was revealed that both insulin and platelet-derived growth factor (PDGF) activate PI3K, and that insulin stimulates GLUT4 translocation to the plasma membrane, while PDGF does not. ${ }^{12}$

Insulin resistance develops as a result of the reduced responsiveness of target tissues to insulin due to decreased receptor and/or downstream signaling protein activity, rather than a reduced circulating insulin level. ${ }^{13,14}$

PI3K/Akt plays a central role in several pathway-related energy homeostases, including leptin, pro-opiomelanocortin (POMC), TNF- $\alpha$, adiponectin, and cytokine stimulation. ${ }^{15-18}$ PI3K consists of a catalytic subunit (p110) and a regulatory subunit $(\mathrm{p} 85 \alpha) .{ }^{5}$ The $\mathrm{p} 85 \alpha$ subunit provides stability and maintains the activity of the $\mathrm{p} 85 \alpha / \mathrm{p} 110$ complex. ${ }^{1,5}$

In addition, the monomer $\mathrm{p} 85 \alpha$ is in competition with the $\mathrm{p} 85 \alpha / \mathrm{p} 110$ complex for binding to IRS1 or allosteric interaction with $\mathrm{p} 110$, provide negative regulation of the p $85 \alpha /$ p110 complex. ${ }^{19}$ The overexpression of $\mathrm{p} 85 \alpha$ by hormonal or experimental induction weakens signal transmission and causes insulin resistance by disrupting the activity of the p $85 \alpha / \mathrm{p} 110$ complex and the connection between PI3K and IRS1. ${ }^{20,21}$
This study found that the SNPs localized in the PIK3R1 gene, encoding $\mathrm{p} 85 \alpha$ regulatory subunit of PI3K which plays a critical role in signal transduction, are associated with T2D, obesity and related phenotypic characteristics. Reports have indicated that SNPs rs706713 (Tyr73Tyr) and rs3730089 (Met326Ile) in exons 1 and 6, respectively, have reduced protein activity and are associated with T2D. ${ }^{22,23}$ Additionally, we also detected a significant association between rs706713 and T2D ( $\mathrm{p}<0.05)$. When the patients were divided into obese and non-obese groups, rs706713 was associated with the disease in the non-obese group $(p=0.05)$. The patient and control groups were in HWE for the SNP rs706713. We also found that this SNP had an effect on HOMA-IR ( $\mathrm{p}=0.018)$ and c-peptide $(\mathrm{p}=0.007)$ levels, although no effect was found on obesity, glucose or insulin levels, a finding that is consistent with a report from Jamshidi et al. ${ }^{23}$ This silent substitution might be interacting with other known and unknown variants.

Many studies have reported conflicting results regarding the association between the Met326Ile (rs3730089) variant in exon 6 of the PIK3R1 gene and insulin resistance and T2D. While this SNP was not found to be a risk factor for diabetes in a Danish population, it was associated with the disease in a Chinese population. ${ }^{24,25}$ This inconsistency in results may be due to differences in sample size and the studied populations. There is evidence that European and Asian populations have different allele frequencies, risk allele profiles, risk genes, and so on. ${ }^{26}$

We also observed a strong association between Met326Ile and the disease $(\mathrm{p}<0.05)$. This missense variation is located between the N-terminal-Src homology region (N-SH2) and the $\mathrm{BH}$ region which has homology with GTPase activating protein (GAP). The $\mathrm{SH} 2$ domains include tyrosine residues and mediate binding to phosphotyrosine residues in the YXXM motif in receptor and adaptor molecules. ${ }^{27}$ Therefore, the Met326Ile variation may alter protein activity by affecting binding to its receptor and adaptor molecules. In our study, the genotype distributions of Met326Ile deviated from HWE in the control groups $(\mathrm{p}<0.05)$. This deviation should be taken into account while assessing the results for Met326Ile. Future studies are needed to reanalyze the results after increasing the sample size to reach equilibrium.

Jamshidi et al. investigated intronic SNPs in the PIK3R1 gene, specifically rs7713645 and rs7709243 in intron 1 and rs1550805 in intron 6, showing that SNPs rs7713645 and rs7709243 were associated with BMI, fasting glucose level and glucose level during the $2^{\text {nd }}$ hour of oral glucose application and that rs1550805 was significantly associated with BMI, body weight and central adiposity. ${ }^{23}$ These results indicate the existence of a strong link between these intronic SNPs, especially rs1550805, and obesity.

In our study, we observed strong associations between the SNPs rs7709243 and rs7713645, and T2Din obese patients $(\mathrm{p}<0.05)$ and between the SNP rs7713645 and T2D in non-obese patients $(\mathrm{p}<0.05)$. In addition, the rs7709243 
variant was determined to have an effect on cholesterol ( $\mathrm{p}=0.008)$ and LDL ( $\mathrm{p}=0.028)$ levels.

On the other hand, we did not detect any association between the intronic SNP rs1550805 and T2D. However, this SNP had an effect on c-peptide $(\mathrm{p}=0.023)$ and BMI $(p=0.007)$. These results support the hypothesis that the intronic SNPs in the PIK3R1 gene have significant effects on BMI and lipid parameters. Because this significant association with T2D was especially observed in the obese group, one could suggest that the rs7709243 and rs7713645 substitutions play a role in the development of T2D through the development of obesity. Additionally, the genotype distributions of these T2D-associated intronic SNPs were in HWE in all groups. Moreover, according to LD analysis, we detected significant linkage only between SNPs rs7709243 and rs7713645 ( $D^{\prime}=0.85$; $\left.\mathrm{r}^{2}=0.7\right)$. Localization at the same intron of both variants might explain this linkage.

Since 2007, more than 60 variants have been shown to be associated with T2D by genome wide association (GWA) analysis, which was performed in numerous populations with large samples. It is noteworthy that the vast majority of these variants are intronic variants. These SNPs do not cause any changes in protein structure but show a strong association with type 2 diabetes, suggesting that they are intronic enhancers. ${ }^{28}$ Additionally, it has been proposed that these variants can be haplotyped with other functional variants. ${ }^{29}$

It has been reported that the SNPs in the noncoding UTR sequence of the PIK3R1 gene, especially SNP rs3756668 in the 3'UTR region, are associated with a high risk of diabetes and elevated BMI as well as increased risk for insulin resistance. ${ }^{30}$ Additionally, the same study revealed that this SNP also has an effect on fasting glucose and fasting insulin levels. ${ }^{30}$ However, our study did not identify a relationship between SNP rs3756668 and T2D or its phenotypes in obese and non-obese individuals in the Turkish population. We suspect that the deviation from HWE in the control group may have influenced our results. Reassessing these results after increasing the population size to reach equilibrium will increase reliability.

Jamshidi et al. investigated the association between the tag SNPs including rs706713, rs7713645, rs7709243, rs251406, rs40318, rs1550805, rs831125 and rs3730089 of the PIK3R1 gene, and BMI, serum leptin level, and glucose/insulin homeostasis in a large population of female twins. ${ }^{23}$ According to their report, rs1550805 in particular was determined to have a strong relationship with serum leptin level and BMI ( $<0.05)$. Consistently, in our study, only rs1550805 was associated with BMI, supporting the findings of Jamshidi et al. ${ }^{23}$

Our study is the $1^{\text {st }}$ to show that SNPs rs706713 (Tyr73Tyr) and rs3730089 (Met326Ile), which are located in exons, and SNPs rs7713645, rs7709243 and rs1550805, which are located in introns, are significantly associated with T2D and have effects on phenotypic features such as obesity, insulin resistance and lipid parameters in a Turkish population. The only exception was SNP rs3756668, which is located in the 3'UTR. Our research findings support the idea that SNPs located in the PIK3R1 gene can affect insulin signaling transduction and thus may play a role in the development of T2D through the development of insulin resistance.

\section{References}

1. Klippel A, Reinhard C, Kavanaugh WM, Apell G, Escobedo MA, Williams LT. Membrane localization of phosphatidylinositol 3-kinase is sufficient to activate multiple signal-transducing kinase pathways. Mol Cell Biol. 1996;16(8):4117-4127.

2. Rojek A, Niedziela M. Insulin receptor and its relationship with different forms of insulin resistance. Adv Cell Biol. 2010;2:59-90.

3. Honardoost M, Sarookhani MR, Arefian E, Soleimani M. Insulin resistance associated genes and miRNA. Appl Biochem Biotechnol. 2014;174(1):63-80.

4. Virtue S, Vidal-Puig A. Adipose tissue expandability, lipotoxicity and the metabolic syndrome - an allostatic perspective. Biochim Biophys Acta. 2010;1801(3):338-349.

5. Yu J, Zhang Y, Mcllroy J, Rordorf-Nikolic T, Orr GA, Backer JM. Regulation of the p85/p110 phosphatidylinositol 3-kinase: Stabilization and inhibition of the p110a catalytic subunit by the $\mathrm{p} 85$ regulatory subunit. Mol Cell Biol. 1998;18(3):1379-1387.

6. Małodobra M, Pilecka A, Gworys B, Adamiec R. Single nucleotide polymorphisms within functional regions of genes implicated in insulin action and association with the insulin resistant phenotype. Mol Cell Biochem. 2011;349(1-2):187-193.

7. Chen JM, Férec C, Cooper DN. A systematic analysis of disease-associated variants in the 30 regulatory regions of human protein-coding genes II: The importance of mRNA secondary structure in assessing the functionality of 30 UTR variants. Hum Genet. 2006;120(3):301-333.

8. Nelsoe RL, Hamid YH, Pociot F, et al. Association of a microsatellite in FASL to type 2 diabetes and of the FAS-670G $>$ A genotype in insulin resistance. Genes Immun. 2006;7:316-321.

9. Gaunt TR, Rodriguez S, Zapata C, Day IN. MIDAS: Software for analysis and visualisation of interallelic disequilibrium between multiallelic markers. BMC Bioinformatics. 2006;27:227. doi: 10.1186/14712105-7-227

10. Kahn CR. The molecular mechanism of insulin action. Annu Rev Med. 1985;36:429-451.

11. Dhe-Paganon S, Ottinger EA, Nolte RT, Eck MJ, Shoelson SE. Crystal structure of the pleckstrin homology-phosphotyrosine binding (PH-PTB) targeting region of insulin receptor substrate 1. Proc Natl Acad Sci. 1999;96(15):8378-8383.

12. Isakoff SJ, Taha C, Rose E, Marcusohn J, Klip A, Skolnik EY. The inability of phosphatidylinositol 3-kinase activation to stimulate GLUT4 translocation indicates additional signaling pathways are required for insulin-stimulated glucose uptake. Proc Natl Acad Sci USA. 1995; 92(22):10247-10251.

13. Perry RJ, Shulman GI. Treating fatty liver and insulin resistance. Aging. 2013;5(11):791-792

14. Knights AJ, Funnell AP, Pearson RC, Crossley M, Bell-Anderson KS. Adipokines and insulin action: A sensitive issue. Adipocyte. 2014; 3(2):88-96.

15. Hotamisligil GS, Peraldi P, Budavari A, Ellis R, White MF, Spiegelman BM. IRS-1-mediated inhibition of insulin receptor tyrosine kinase activity in TNF-alpha- and obesity-induced insulin resistance. Science.1996; 271(5249):665-668.

16. Bogan JS, Lodish HF. Two compartments for insulin-stimulated exocytosis in 3T3-L1 adipocytes defined by endogenous ACRP30 and GLUT4. J Cell Biol. 1999;146(3):609-620.

17. Chagnon YC, Rankinen T, Snyder EE, Weisnagel SJ, Pe'russe L, Bouchard C. The human obesity gene map: The 2002 update. Obes Res. 2003;11(3):313-367.

18. Bokarewa M, Nagaev I, Dahlberg L, Smith U, Tarkowski A. Resistin, an adipokine with potent proinflammatory properties. J Immunol. 2005;174(9):5789-5795. 
19. Ueki K, Yballe CM, Brachmann SM, et al. Increased insulin sensitivity in mice lacking $\mathrm{p} 85$ beta subunit of phosphoinositide 3-kinase. PNAS. 2002;99(1):419-424.

20. Barbour LA, Shao J, Qiao L, et al. Human placental growth hormone increases expression of the $\mathrm{P} 85$ regulatory unit of phosphatidylinositol 3-kinase and triggers severe insulin resistance in skeletal muscle. Endocrinology. 2004;145(3):1144-1150.

21. Giorgino F, Pedrini MT, Matera L, Smith RJ. Specific increase in p85a expression in response to dexamethasone is associated with inhibition of insulin-like growth factor-1 stimulated phosphatidylinositol 3-kinase activity in cultured muscle cells. J Biol Chem. 1997;272(11):7455-7463.

22. Rai E, Sharma S, Kaul S, et al. The interactive effect of SIRT1 promoter region polymorphism on type 2 diabetes susceptibility in the north Indian population. Plos One. 2012;7(11):e48621. doi: 10.1371/journal. pone.0048621

23. Jamshidi Y, Snieder $\mathrm{H}$, Wang $\mathrm{X}$, et al. Phosphatidylinositol 3-kinase $\mathrm{p} 85$ alpha regulatory subunit gene PIK3R1 haplotype is associated with body fat and serum leptin in a female twin population. Diabetologia. 2006;49(11):2659-2667.

24. Hansen $L$, Zethelius $B$, Berglund $L$, et al. In vitro and in vivo studies of a naturally occurring variant of the human p85a regulatory subunit of the phosphoinositide 3-kinase. Inhibition of protein kinase $b$ and relationships with type 2 diabetes, insulin secretion, glucose disappearance constant, and insulin sensitivity. Diabetes. 2001;50:691-693.
25. Chen S, Yan W, Huang J, Ge D, Yao Z. Association analysis of the variant in the regulatory subunit of phosphoinositide 3-kinase (p85a) with type 2 diabetes mellitus and hypertension in the Chinese Han population. Diabet Med. 2005;22(6):737-743.

26. Hancock AM, Witonsky DB, Gordon AS, et al. Adaptations to climate in candidate genes for common metabolic disorders. PLoS Genet. 2008;4(2):e32. doi: 10.1371/journal.pgen.0040032

27. Fruman DA, Cantley LC. Phosphoinositide 3-kinase in immunological systems. Semin Immunol. 2002;14(1):7-18.

28. Pang DX, Smith AJP, Humphries SE. Functional analysis of TCF7L2 genetic variants associated with type 2 diabetes. Nutr Metab Cardiovasc Dis. 2013;23(6):550-556.

29. Hossain S, Islam S, Khatun T, Sapon A. Type 2 diabetes mellitus: Impact of genetics and environment. Int J Curr Sci. 2014;12:110-127.

30. Małodobra $M$. The role of single nucleotide polymorphisms of untranslated regions (Utrs) in insulin resistance pathogenesis in patients with type 2 diabetes. In: Croniger C, ed. Medical Complications of Type 2 Diabetes. London, UK: InTech; 2011:165-188. 